\title{
Applying the Drucker-Prager Failure Criterion for Representing Soil Behavior using Smoothed Particle Hydrodynamics
}

\author{
Erly Bahsan ${ }^{1 *}$, Bunyamin Andreatama1, Widjojo A. Prakoso ${ }^{1}$, Budi Susilo Soepandji1, \\ R.R. Dwinanti R. Marthanty ${ }^{1}$ \\ ${ }^{1}$ Department of Civil and Environmental Engineering, Faculty of Engineering, Universitas Indonesia, \\ Kampus UI Depok, Depok 16424, Indonesia
}

\begin{abstract}
Smoothed Particle Hydrodynamics (SPH) has previously used in hydrodynamics as a Lagrangian numerical method to simulate fluid behavior. Recently the SPH is also being used to simulate soil deformation, since the largely known Finite Element Method (FEM) cannot represent the soil deformation after failure. In SPH, soil materials are modeled as a set of particles, and the behavior of those particles can be simplified using the equivalent viscosity or using the soil failure criterion. This research tries to implement the Drucker-Prager model using Fortran platform to simulate a simple soil model deformation behavior, by modifying the previous model that used the equivalent viscosity. A simple model of small slope is built by a stack of SPH particles, which are expected to behave as the collapsing soil mass. The movement of the particles and the change of the model's geometry are observed visually after the SPH simulation. The preliminary results show that the particles can already behave like a failure in granular soil, yet it still needs to be improved due to the unwanted particle movements.
\end{abstract}

Keywords: $\quad$ Fortran; Numerical method; Smoothed particle hydrodynamics; Soil constitutive model

\section{Introduction}

The most popular numerical method used in geotechnical analyses nowadays is the Finite Element and Finite Difference. Those methods have the ability of simulating the stress-strain behaviour of soil, hence they can predict the deformations. However, both numerical methods have drawback regarding the large deformation or the deformation after failure due to the mesh and grid system. To overcome this problem, the Smoothed Particle Hydrodynamics had been introduced.

Smoothed Particle Hydrodynamics (SPH) is a mesh-free or gridless particle method based on Lagrangian formulation, that was first applied to solve the astrophysical problems in open space (e.g., Gingold and Monaghan, 1977; Lucy, 1977). Recently, this method is also applied for geomaterials and geodisaster models as mentioned by Huang et al. (2014) and Bui and Nguyen (2021). Examples of applications are for dam-break analysis (Wang and Shen, 1999), large deformation and slope failure (Bui et al., 2011), seepage (Maeda et al., 2004), piping erosion (Sjah and Vincent, 2017), liquefaction and flow failure (Naili et al., 2005; El Shamy and Sizkow, 2021; Sizkow and El Shamy, 2021). 
This article presents the progress of the on-going research on the use of SPH model to simulate the soil deformation after failure. The large lateral deformation phenomenon after the big earthquake in Central Sulawesi, Indonesia, in 2018 highlights the importance of the prediction of post failure deformation. The SPH research group in the Department of Civil and Environmental engineering of University of Indonesia tried to develop their own platform to model the post-liquefaction deformation. The first attempt has been conducted using the equivalent viscosity as in (Mahardima et al., 2021). The liquefied soil behaviour was modelled as Bingham fluid, which is one of the viscoplastic models (e.g., Uzuoka et al., (1998).

In this article, the Elasto-plastic SPH Procedure is used in its programming algorithm with the Drucker-Prager model to describe the behaviour of soil particles which refers to research that has been developed by Bui et al. (2008). The Drucker-Prager failure criterion is one of the soil constitutive law to represent the elasto-plastic soil behaviour, and is considered as the most stable compared to the other criterion (e.g., Potts and Zdravković, 2001). The recent model is built base on the previous codes by Mahardima et al. (2021) using Fortran platform. This study is a small part of the bigger research scheme with the main objective is to build a SPH model to predict the large deformation after liquefaction.

\section{Theoretical Base}

\subsection{Basic Concept of SPH}

The concept of SPH as mentioned by Liu and Liu (2010) is that a state of a system is represented by a set of particles, which possess the material properties and interact with each other within a range that controlled by a weight or smoothing function. SPH is built on interpolation theory with two essential aspects, which are the kernel approximation and particle approximation (e.g., Huang et al., 2014). Consider a particle $\alpha$ as shown in Figure 1, the value of a function at computing particle $\alpha$ is approximated using the average of those values of the function at all particles in the influence domain of particle $\alpha$, weighted by the smoothing function, which is $\mathrm{W}$ in Figure 1. The neighbouring particle is shown as $\beta$, and the radius of influence from particle $\alpha$ is $\mathrm{h}$.

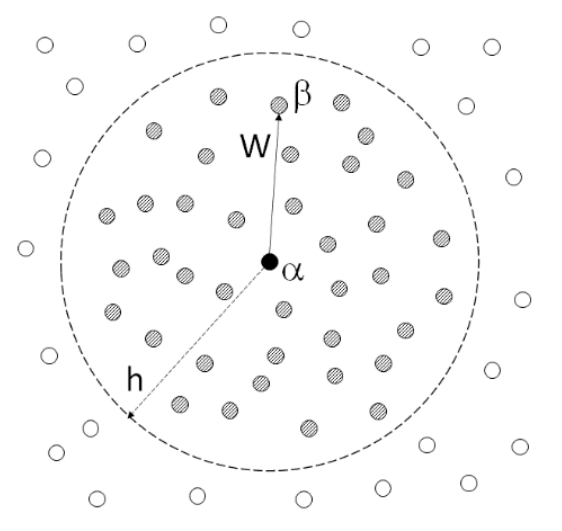

Figure 1 Basic Concept of SPH

The kernel approximation can be written as a function $\mathrm{f}(\mathrm{x})$ as the position of vector $\mathrm{x}$ :

$$
\langle f(x)\rangle=\int_{\Omega} f\left(x^{\prime}\right) W\left(x-x^{\prime}, h\right) d x^{\prime}
$$

where $\Omega$ is the volume of integral that contains $\mathrm{x}$. The particle approximation can be written as: 


$$
\langle f(x)\rangle=\sum_{j=1}^{N} \frac{m_{j}}{\rho_{j}} f\left(x_{j}\right) W\left(x-x_{j}, h\right)
$$

where $\mathrm{N}$ is the total number of particles within the influence area of the particle at $\mathrm{x}$, and $\mathrm{m} / \rho$ is the ratio of mass to the density of particle $\mathrm{j}$. There are some alternatives of smoothing function $\mathrm{W}$, one of them as suggested in Bui et al. (2008) is written as:

$$
W_{i j}=\left\{\begin{array}{cc}
\frac{5}{14 \pi h^{5}}\left(4 h^{3}-6 r^{2} h+3 r^{3}\right), & 0 \leq r / h<1 \\
\frac{5}{14 \pi h^{5}}(2 h-r)^{3}, & 1 \leq r / h<2 \\
0, & r / h \geq 2
\end{array}\right.
$$

\subsection{Governing Equation}

Elasto-plastic SPH model follows the governing equation that consists of the conservation of mass and conservation of momentum. The continuity equation is written as:

$$
\frac{\partial \rho}{\partial t}=-\rho \frac{\partial v^{\alpha}}{\partial x^{\alpha}}
$$

where $\rho$ is density, $\mathrm{t}$ is time, $\mathrm{v}$ represents the velocity vector. The ground motion equation, which is based on conservation of momentum is:

$$
\frac{\partial v^{\alpha}}{\partial t}=\frac{1}{\rho} \frac{\partial \sigma^{\alpha \beta}}{\partial x^{\alpha}}+F
$$

where $\alpha$ and $\beta$ are Cartesian components $\mathrm{x}, \mathrm{y}$ and $\mathrm{z} ; \sigma$ is the total stress tensor and $\mathrm{F}$ external force vector which is the gravitational force in this study. The Equations 4 and 5 can be written in $\mathrm{SPH}$, respectively as:

$$
\begin{aligned}
& \frac{D \rho}{D t}=-\sum_{\beta}^{N} m_{j}\left(v_{i}^{\alpha}-v_{i}^{\alpha}\right) \frac{\partial W_{i j}}{\partial x_{j}^{\alpha}} \\
& \frac{D v_{i}^{\alpha}}{D t}=\sum_{j=1}^{N} m_{j}\left(\frac{\sigma_{i}^{\alpha \beta}}{\rho_{i}^{2}}+\frac{\sigma_{j}^{\alpha \beta}}{\rho_{j}^{2}}-\delta^{\alpha \beta} \Pi_{i j}\right) \frac{\partial W_{i j}}{\partial x_{j}^{\beta}}+g^{\alpha}
\end{aligned}
$$

where $\delta^{\alpha \beta}$ is Kronecker's delta which has a value of $\delta^{\alpha \beta}=1$ if $\alpha=\beta$, and $\delta^{\alpha \beta}=0$ if $\alpha \neq \beta$.

\subsection{Soil Constitutive Model}

The soil behavior is simplified using the elastic-perfectly plastic model. This model is presented by the total strain rate tensor, $\varepsilon^{\cdot \alpha \beta}$, which consists of the elastic strain tensor $\varepsilon_{e}^{\cdot \alpha \beta}$ and plastic strain tensor $\varepsilon_{p}^{. \alpha \beta}$. Each of those strain tensor is described as the following:

$$
\begin{aligned}
& \dot{\varepsilon}_{e}^{\alpha \beta}=\frac{\dot{s}^{\alpha \beta}}{2 G}+\frac{1-2 v}{3 E} \dot{\sigma}^{\gamma \gamma} \delta^{\alpha \beta} \\
& \dot{\varepsilon}_{p}^{\alpha \beta}=\dot{\lambda} \frac{\partial g}{\partial \sigma^{\alpha \beta}}
\end{aligned}
$$

where $s^{\cdot \alpha \beta}$ is the deviatoric shear stress rate tensor, $v$ is Poisson's ratio, $\mathrm{G}$ is the shear modulus, $\mathrm{E}$ is Young's modulus, $\sigma^{\cdot \gamma \gamma}$ is the sum of the three components of the normal stress, $\lambda^{\cdot}$ is the rate of change of the plastic multiplier $\lambda$, and $g$ is the plastic potential function.

In this study, the Drucker-Prager failure criterion is chosen as to represent the soil plastic flow behavior. Drucker-Prager criterion was used in the previous study by Bui et al. 
(2008). It is known that this failure criterion improved the Mohr-Coulomb criterion as it seen on Figure 2, that the sphere shape of Drucker-Prager covers the hexagonal shape of Mohr-Coulomb. This condition is written as:

$$
\begin{aligned}
& f\left(I_{1}, J_{2}\right)=\sqrt{J_{2}}+\alpha_{\phi} I_{1}-k_{c}=0 \\
& I_{1}=\sigma^{x x}+\sigma^{y y}+\sigma^{z z} \text { and } J_{2}=\frac{1}{2} \dot{s}^{\alpha \beta} \dot{s}^{\alpha \beta} \\
& \alpha_{\phi}=\frac{\tan \phi}{\sqrt{9+12 \tan ^{2} \phi}} \text { and } k_{c}=\frac{3 c}{\sqrt{9+12 \tan ^{2} \phi}}
\end{aligned}
$$

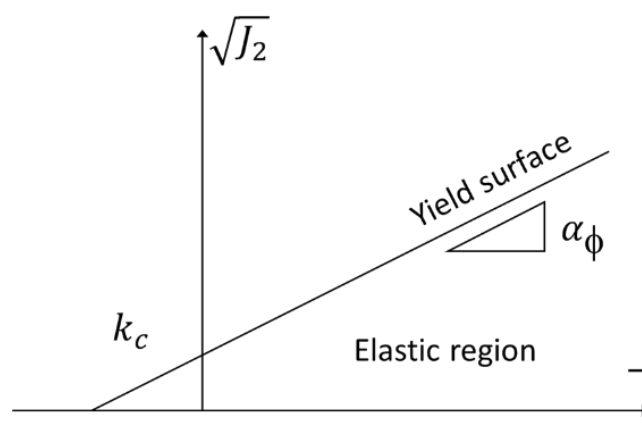

Failure criterion in $\left(-I_{1}, \sqrt{J_{2}}\right)$ plane

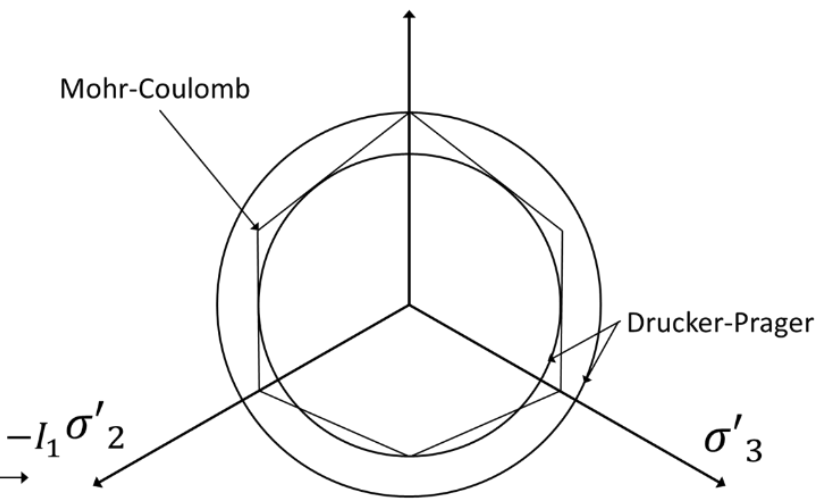

Shape of failure criterion in $\pi$ plane

Figure 2 Illustration of Drucker-Prager criterion (reproduced from Bui et al., 2008)

where $\alpha^{\phi}$ and $k_{c}$ are Drucker-Prager constants, which correspond to the Coulomb material constants $\mathrm{c}$ (cohesion) and $\phi$ (angle of friction); $\sigma^{x x}, \sigma^{y y}, \sigma^{z z}$ are three normal stress components; $I_{1}$ and $J_{2}$ are the first and second invariants of the stress tensor. The plastic potential function is chosen to be similar as the yield function (associated flow rule) of Drucker-Prager, thus it can be expressed as:

$$
g=\sqrt{J_{2}}+\alpha_{\phi} I_{1}-k_{c}
$$

To determine the second invariants $J_{2}$ for the Drucker-Prager criterion, the deviatoric shear stress rate $s^{\cdot \alpha \beta}$ should be obtained from the rheological model as described in Yang et al. (2020) as:

$$
\begin{aligned}
& \dot{s}^{\alpha \beta}=2 \eta \dot{\varepsilon}^{\alpha \beta} \\
& \eta=\frac{\mu P}{\sqrt{\dot{\varepsilon}^{\alpha \beta} \dot{\varepsilon}^{\alpha \beta}}} \\
& \mu=\mu_{s}+\frac{\mu_{p}-\mu_{s}}{I_{0} / I_{i}+1} \\
& P_{i}=c^{2}\left(\rho_{i}-\rho_{0}\right)
\end{aligned}
$$

where $\eta$ is the apparent viscosity, $\mathrm{P}$ is the isotropic pressure, $\mathrm{c}$ is the speed of sound, $\rho_{i}$ is the density of particle $\mathrm{i}, \rho_{0}$ is the reference density of the material. The plastic multiplier $\lambda$. for the associated flow rule is calculated from the Drucker-Prager constants as:

$$
\dot{\lambda}=\frac{3 \alpha_{\phi} K \dot{\varepsilon}^{\gamma \gamma}+\left(G / \sqrt{J_{2}}\right) s^{\alpha \beta} \dot{\varepsilon}^{\alpha \beta}}{9 \alpha_{\phi}^{2} K+G}
$$


The rate of stress that is invariant to the rigid-body rotation should be employed for constitutive relations considering the large deformation. For this purpose, Bui et al. (2008) use the Jaumann stress rate:

$$
\dot{\hat{\sigma}}^{\alpha \beta}=\dot{\sigma}^{\alpha \beta}-\sigma^{\alpha \gamma} \dot{\omega}^{\beta \gamma}-\sigma^{\gamma \beta} \dot{\omega}^{\alpha \gamma}
$$

where $\dot{\omega}$ is spin rate tensor which defined as:

$$
\dot{\omega}^{\alpha \beta}=\frac{1}{2}\left(\frac{\partial v^{\alpha}}{\partial x^{\beta}}-\frac{\partial v^{\beta}}{\partial x^{\alpha}}\right)
$$

The above-mentioned equations are included in the algorithm for numerical calculation which will be translated to Fortran codes.

\section{Methodology}

The steps in this study are first building the algorithm for numerical iteration based on the related equations, followed by translating the equations to the Fortran codes based on the algorithm. After that the codes are executed and the output are plotted so they can be evaluated visually.

\subsection{Algorithm for Numerical Calculation}

The numerical calculation in this study is carried out using Fortran platform. The algorithm for the simulation is shown as flow chart in Figure 3.

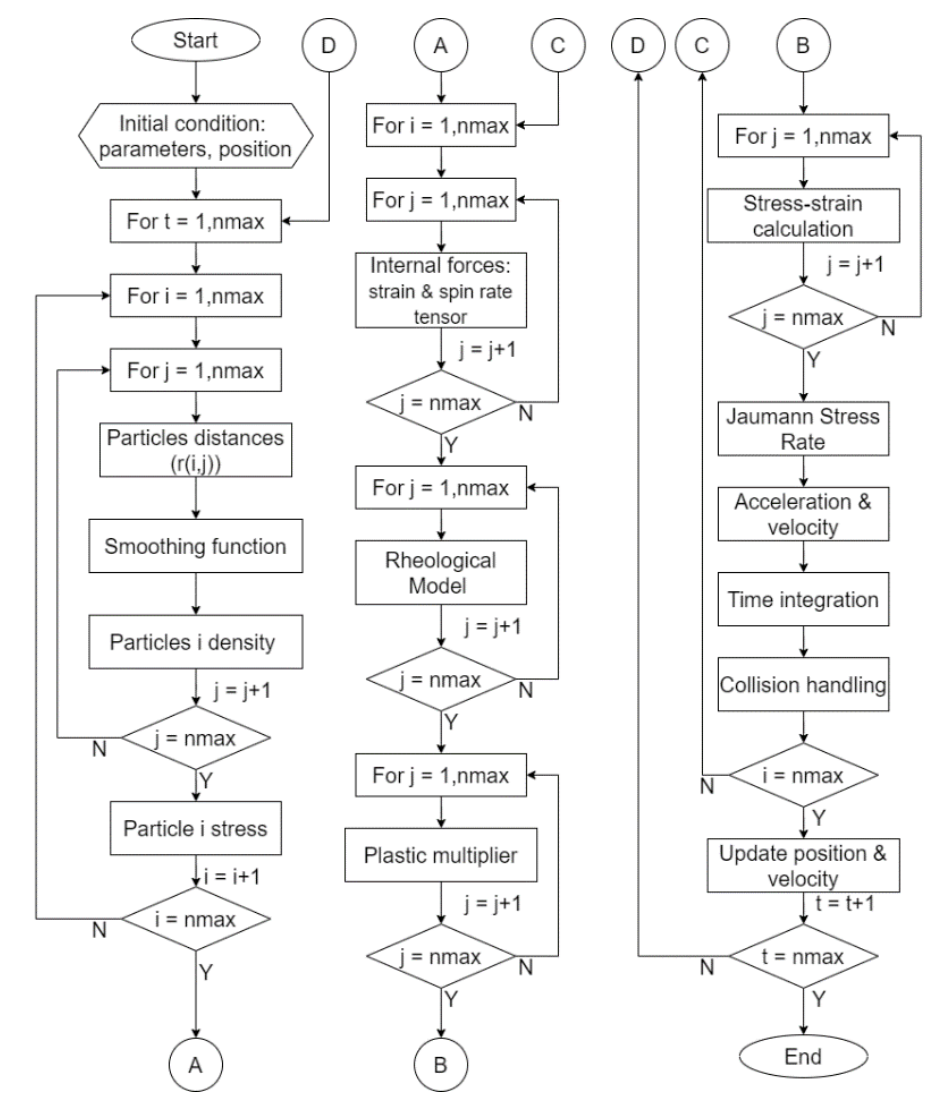

Figure 3 Flowchart for the SPH simulation algorithm

The steps begin with stating the initial conditions and positions of the particles, then it will do an iteration for each time steps, which includes the smoothing function that governs the behavior of each particle to the neighboring particles as written in Equation 3. The 
implementation of Drucker-Prager failure criterion which are spread out in Equation 8 to Equation 20 are included in the iterations of rheological model, plastic multiplier, stressstrain calculation, and Jaumann stress rate. After that, the process continues with acceleration and velocity updates for each particle, time integration, and collision handling. The final step is for updating the position and velocity of each particle in each iteration. Some examples of the written codes will be shown in the following section.

\section{Results}

\subsection{Numerical Simulation}

As mentioned in the previous section, the simulation begins with the declaration of initial position and velocity of the particles. The example of Fortran codes for this step is:

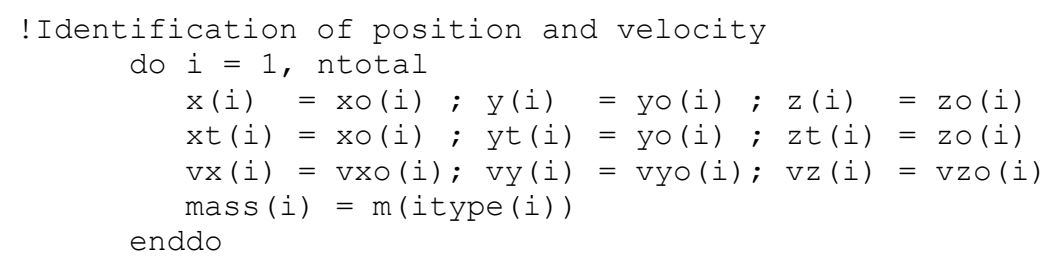

These particles are arranged in a 3D planes, with the shape resembles a small slopes as shown in Figure 4. The material is assigned to be a loose soil with cohesion $10 \mathrm{kPa}$ and angle of friction $20^{\circ}$ (Andreatama, 2021)
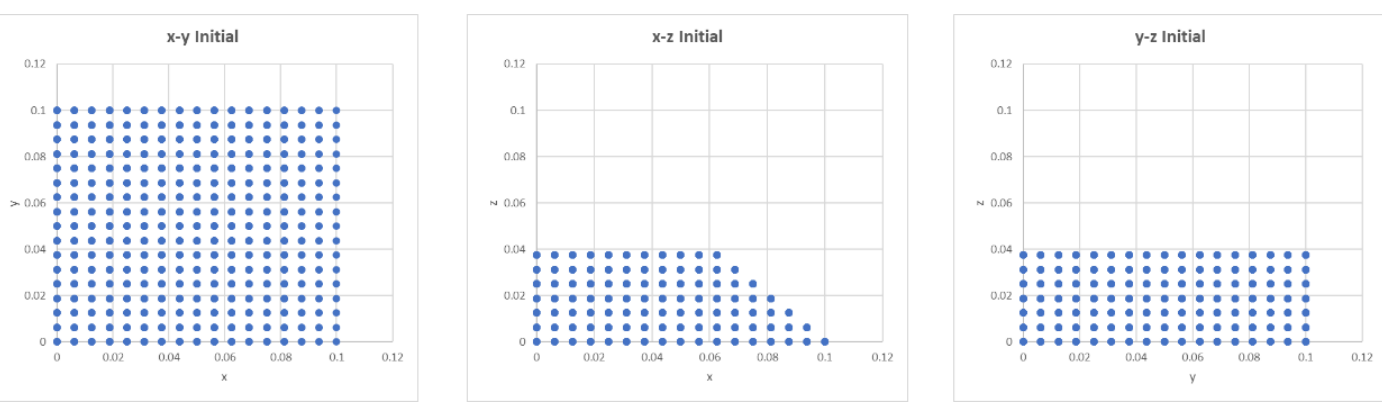

Figure 4 The intial condition of particles with the view from the top ( $x-y$ plane), side ( $x-z$ plane), and front ( $y-z$ plane)

The soil parameters such as cohesion and angle of friction are included in the Drucker-Prager constants as in Equation (10), and written in the codes as:

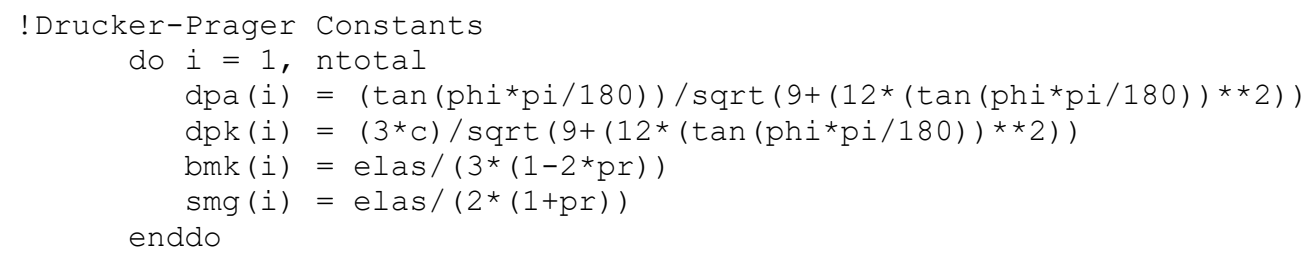

The density of particles is calculated after the calculation of distances between particles through the smoothing kernel, as written in the codes:

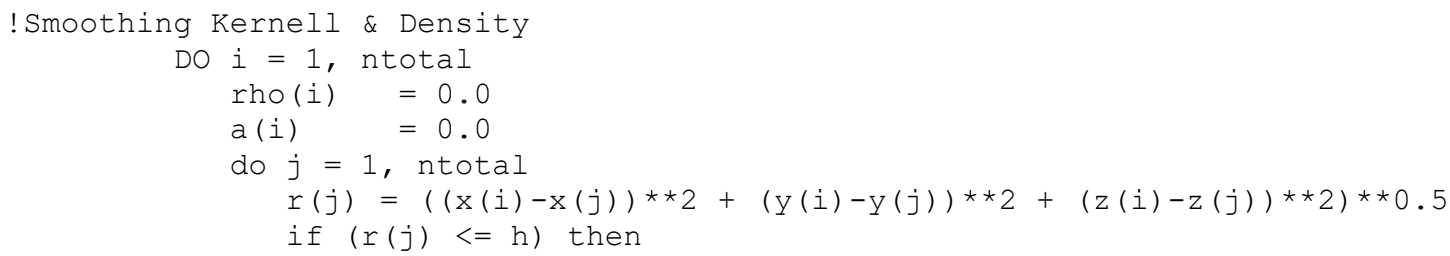




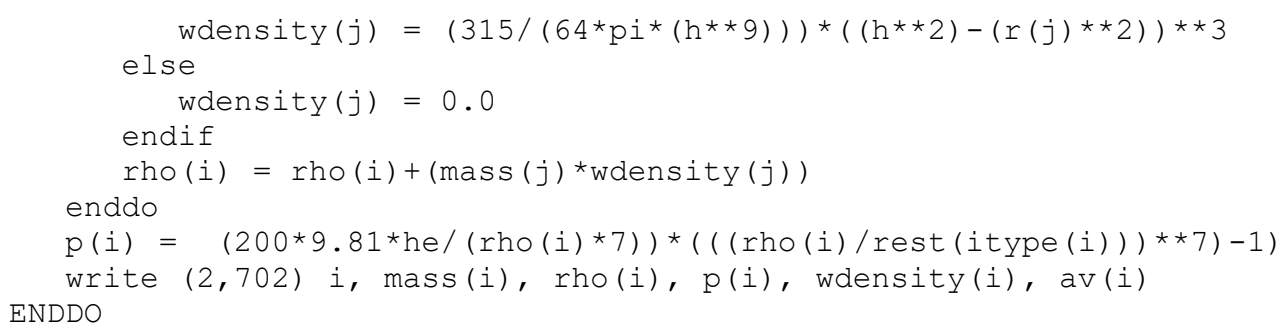

The strain rate which related to the particle velocity is calculated through these codes:

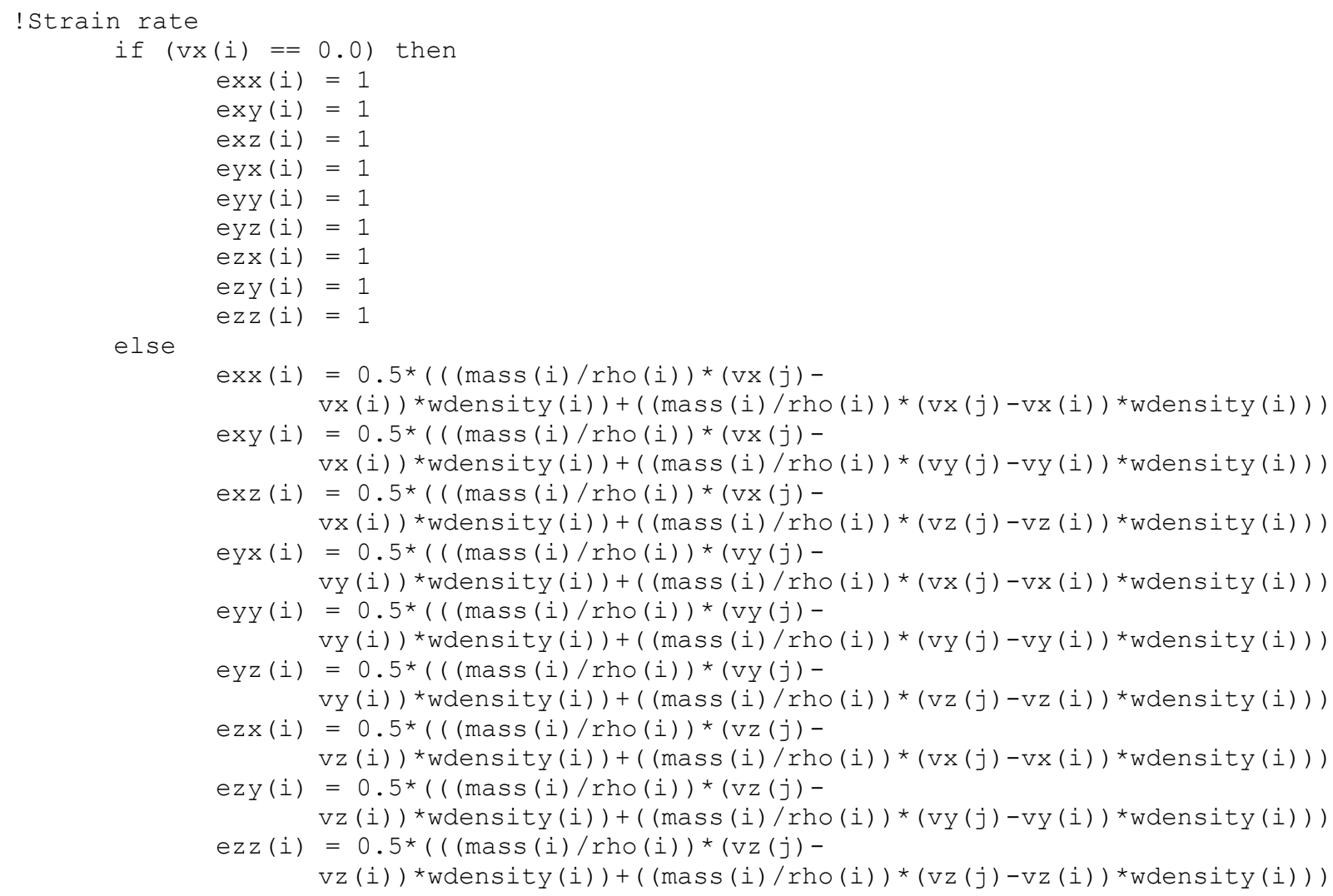

To obtain the particle stress, the plastic multiplier (Equation 18) should be calculated first from the Drucker-Prager constants as written in these codes:

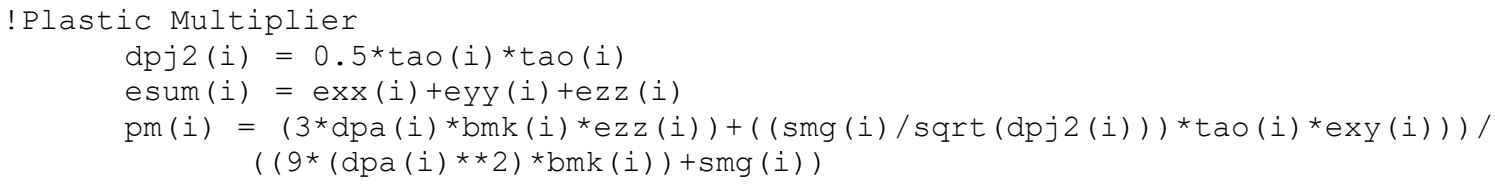

The last parts of the codes represent the steps for updating collision handling, which resulting to the updated positions of each particle.

\section{Discussion}

The results of the above simulation are the position of each particle relative to the 3D coordinate system for each iteration step. The examples of the visualized results are shown here in Figure 5, Figure 6, Figure 7, for the $10^{\text {th }}, 50^{\text {th }}$, and $100^{\text {th }}$ iterations, respectively. 

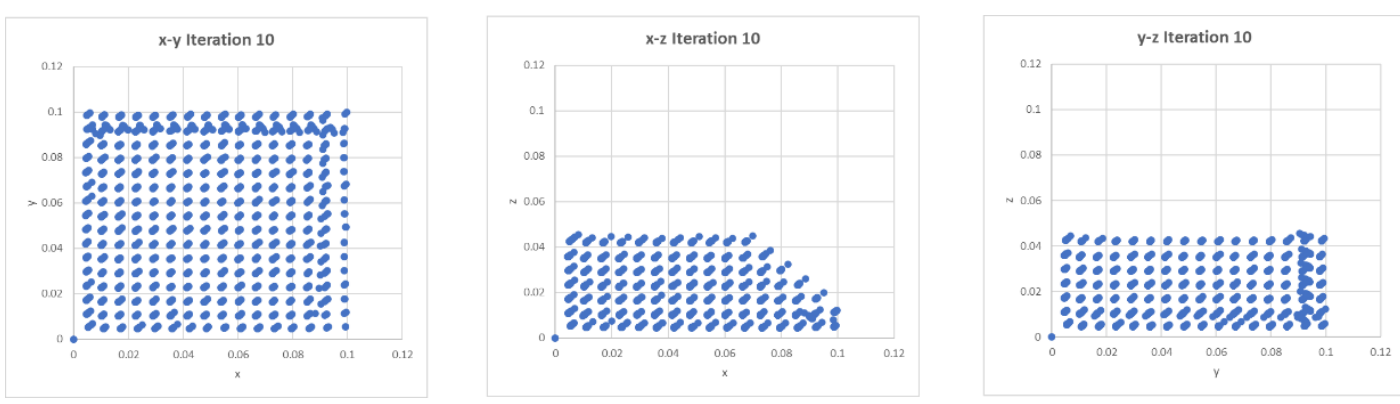

Figure 5 The particles' position after $10^{\text {th }}$ iteration
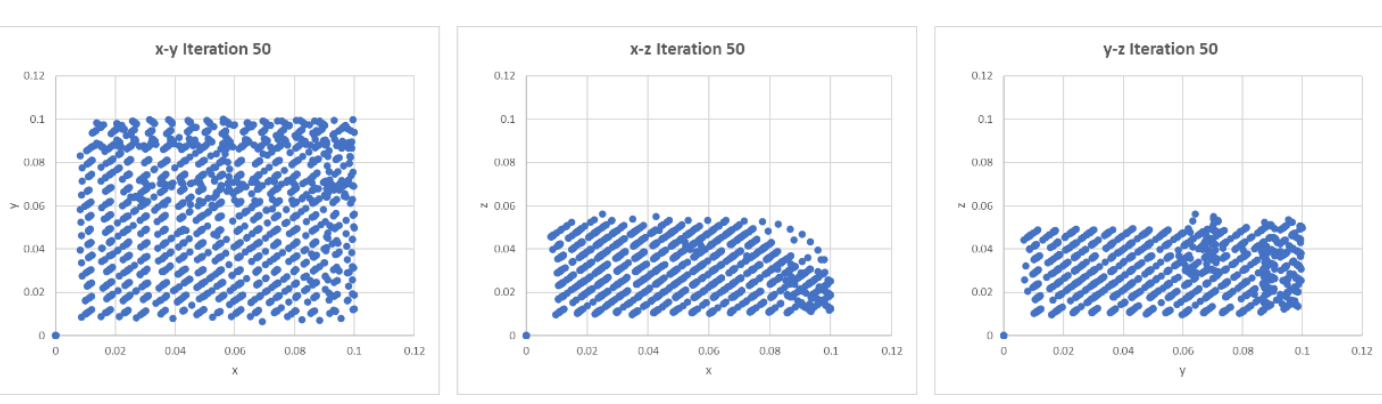

Figure 6 The particles' position after $50^{\text {th }}$ iteration
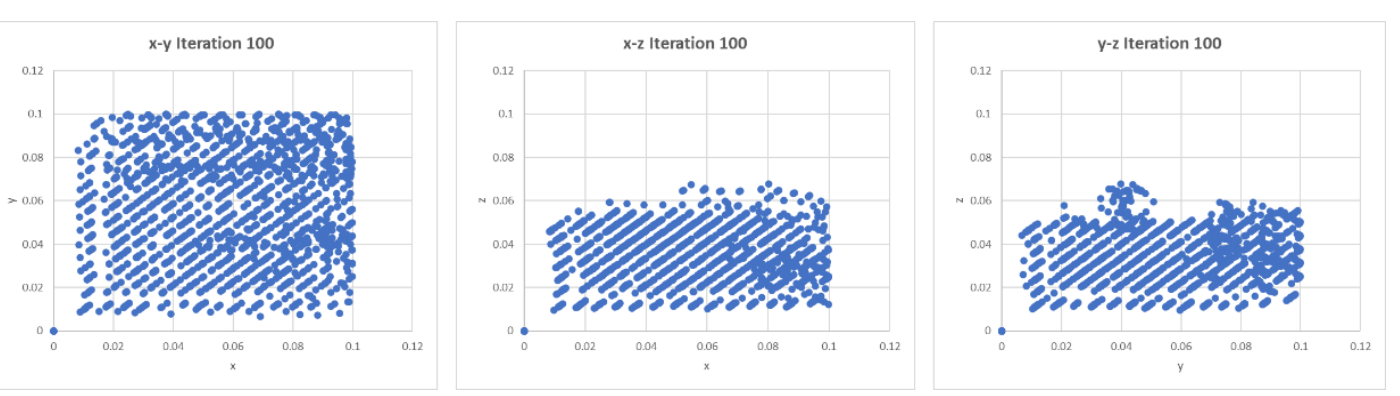

Figure 7 The particles' position after $100^{\text {th }}$ iteration

It is shown that the movement of particle is visually resemble the behavior of loose granular materials at the $10^{\text {th }}$ iteration and $50^{\text {th }}$ iteration. However, the $100^{\text {th }}$ iteration shows an unexpected behavior where the particles still jumping up instead of staying in the more stable position. Another anomaly also shown near the boundary of the sample, where the particles tend to avoid the initial boundary. The cause of this behavior is still unknown and needs to be address in the next stage of research. This problem was similarly found in the previous research by Mahardima et al. (2021) in which the model uses equivalent viscosity instead of the soil constitutive model. Hence, the problem may not be caused by the choice between the application of equivalent viscosity or soil constitutive model such as Drucker-Prager criterion.

\section{Conclusions}

This article shows the progress of an on-going research of application of SPH method to the simulation of granular materials using the Fortran platform. The main objective is to model the behavior of granular materials (i.e., sands) while interacting with water during the liquefaction phenomena. At the recent study, the results have shown that the proposed algorithm can simulate the behavior of granular material as particles after the failure. Yet the interaction between particles still not completely correct, possibly due to the later part 
of the algorithm, which is the collision handling. This part will be the next subject for the next study.

\section{Acknowledgements}

The authors of this article want to acknowledge the SPH Research Group at the Department of Civil Engineering University of Indonesia for the helps and discussions during this study. This research was also supported partially by The Ministry of Education, Culture, Research, and Technology through the WCR Research Grant NKB389/UN2.RST/HKP.05.00/2021.

\section{References}

Andreatama, B., 2021. Permodelan Perilaku Partikel Tanah Kohesif Pada Stabilitas Lereng Menggunakan Metode Smoothed Particle Hydrodynamics (SPH). Modeling Cohesive Soil Particle Behavior on Slope Stability using Smoothed Particle Hydrodynamics Method. Master's Thesis, Graduate Program, Universitas Indonesia, Depok

Bui, H.H., Fukagawa, R., Sako, K., Ohno, S., 2008. Lagrangian Meshfree Particles Method (SPH) for Large Deformation and Failure Flows of Geomaterial using Elastic-Plastic Soil Constitutive Model. International Journal for Numerical and Analytical Methods in Geomechanics, Volume 32(12), pp. 1537-1570

Bui, H.H., Fukagawa, R., Sako, K., Wells, J.C., 2011. Slope Stability Analysis and Discontinuous Slope Failure Simulation by Elasto-Plastic Smoothed Particle Hydrodynamics (SPH). Geotechnique, Volume 61(7), pp. 565-574

Bui, H.H., Nguyen, G.D., 2021. Smoothed Particle Hydrodynamics (SPH) and its Applications in Geomechanics: From Solid Fracture to Granular Behavior and Multiphase Flows in Porous Media. Computers and Geotechnics, Volume 138, https://doi.org/10.1016/j.compgeo.2021.104315

El Shamy, U., Sizkow, S.F., 2021. Coupled SPH-DEM Simulations of Liquefaction-Induced Flow Failure. Soil Dynamics and Earthquake Engineering, Volume 144, https://doi.org/10.1016/j.soildyn.2021.106683

Gingold, R.A., Monaghan, J.J., 1977. Smoothed Particle Hydrodynamics: Theory and Application to Non-Spherical Stars. Monthly Notices of the Royal Astronomical Society, Volume 181(3), pp. 375-389

Huang, Y., Dai, Z., Zhang, W., 2014. Geo-disaster Modeling and Analysis: An SPH-based Approach. Switzerland: Springer

Liu, M.B., Liu, G.R., 2010. Smoothed Particle Hydrodynamics (SPH): An Overview and Recent Developments. Archives of Computational Methods in Engineering, Volume 17, pp. 2576

Lucy, L.B., 1977. A Numerical Approach to the Testing of the Fission Hypothesis. Astronomical Journal, Volume 82, pp. 1013-1024

Maeda, K., Sakai, H., Sakai, M., 2004. Development of Seepage Failure Analysis Procedure of Granular Ground with Smoothed Particle Hydrodynamics (SPH) Method. Structural Engineering/ Earthquake Engineering, Volume 23(2), pp. 307s-319s

Mahardima, H., Bahsan, E., Marthanty, R.R.D.R., 2021. Development of Smoothed Particle Hydrodynamics (SPH) Method to Model the Interaction of Sand and Water During Liquefaction with Bingham Fluid Model Adaptation. IOP Conference Series: Earth and Environmental Science, Volume 622, pp. 1-15 
Naili, M., Matsushima, T., Yamada, Y., 2005. A 2D Smoothed Particle Hydrodynamics Method for Liquefaction Induced Lateral Spreading Analysis. Journal of Applied Mechanics, Volume 8, pp. 591-599

Potts, D.M., Zdravković, L., 2001. Finite Element Analysis in Geotechnical Engineering: Volume Two - Application. ICE Publishing, Thomas Telford

Sizkow, S.F., El Shamy, U., 2021. SPH-DEM Simulations of Saturated Granular Soils Liquefaction Incorporating Particles of Irregular Shape. Computers and Geotechnics Volume 134, https://doi.org/10.1016/j.compgeo.2021.104060

Sjah, J., Vincent, E., 2017. Fluid-Solid Interaction in the Case of Piping Erosion: Validation of A SPH-ALE Code. International Journal of Technology, Volume 8(6), pp. 1040-1049

Uzuoka, R., Yashima, A., Kawakami, T., Konrad, J.-M., 1998. Fluid Dynamics Based Prediction of Liquefaction Induced Lateral Spreading. Computers and Geotechnics, Volume 22, pp. $243-282$

Wang, Z., Shen, H.T., 1999. Lagrangian Simulation of One-Dimensional Dam-Break Flow. Journal of Hydraulic Engineering, Volume 125(11), pp. 1217-1220

Yang, E., Bui, H.H., De Sterck, H., Nguyen, G.D., Bouazza, A., 2020. A Scalable Parallel Computing SPH Framework for Predictions of Geophysical Granular Flows. Computers and Geotechnics, Volume 121, https://doi.org/10.1016/j.compgeo.2020.103474 